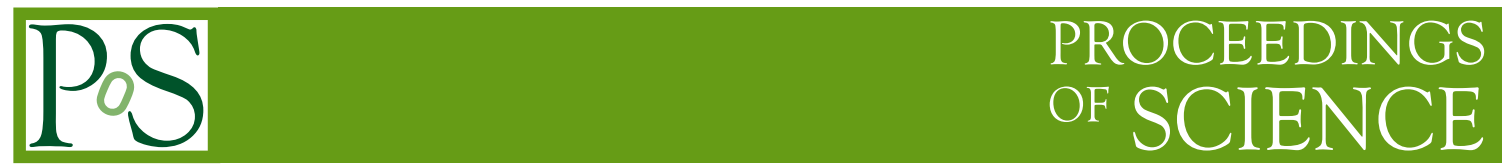

\title{
Searches for BSM Higgs bosons at ATLAS and CMS
}

\author{
Adam Bailey $^{a, 1, *}$, on behalf of the ATLAS and CMS collaborations \\ ${ }^{a}$ Instituto de Fisica Corpuscular (IFIC), Centro Mixto Universidad de Valencia - CSIC \\ Spain \\ E-mail: adam.bailey@cern.ch
}

Several beyond-the-Standard-Model (BSM) theories predict an extended Higgs sector, which motivates searches for neutral and charged Higgs bosons in addition to the Higgs boson already observed at $125 \mathrm{GeV}$. Run 2 of the LHC was completed in 2018 and provided a total of $139 \mathrm{fb}^{-1}$ of data giving an opportunity to further explore the BSM Higgs parameter space. This proceeding discusses searches for BSM Higgs bosons at the ATLAS and CMS experiments with either the early $\left(36 \mathrm{fb}^{-1}\right)$ or full LHC Run 2 dataset.

The Eighth Annual Conference on Large Hadron Collider Physics-LHCP2020

25-30 May, 2020

online

\footnotetext{
${ }^{1}$ The author acknowledges the support from project RTI2018-094270-B-I00, Spanish Ministry of Innovation and Research and ERDF.

${ }^{*}$ Speaker
} 


\section{Introduction}

Several beyond-the-Standard-Model (BSM) theories predict an extended Higgs sector. Typically these are two Higgs doublet models (2HDM) [1], which predict a total of five Higgs bosons: two neutral CP even $(h, H)$, one neutral CP odd $(A)$, and two charged $\left(H^{ \pm}\right)$. A well-known 2HDM is the Minimal Supersymmetric Model (MSSM) [2]. The MSSM scenario can be described at the tree level in terms of the mass of the CP-odd neutral Higgs $\left(m_{A}\right)$ and the ratio of the vacuum expectation values of the two Higgs doublets $(\tan \beta)$. hMSSM [3, 4] and $m_{h}^{\bmod }$ [5] are commonly used as benchmark scenarios, but new $M_{h}^{125}$ benchmarks [6] were proposed in 2019, which were designed to be compatible with the Run 2 results up until that point. Analyses set model-independent limits on the Higgs boson production cross section times branching ratio and interpret results for particular MSSM models in the $m_{A}$-tan $\beta$ plane.

Any BSM model must be compatible with the existence of the neutral $125 \mathrm{GeV}$ Higgs boson discovered in 2012 [7, 8]. The additional neutral Higgs bosons can either be lighter or heavier than $125 \mathrm{GeV}$ and searches are carried out for both cases.

Run 2 of the LHC data taking was completed at the end of 2018. Many analyses using the ATLAS [9] and CMS [10] experiments have results based on a partial Run 2 dataset, corresponding to $36 \mathrm{fb}^{-1}$. The complete dataset of $139 \mathrm{fb}^{-1}$ is now available. Some analyses already use this dataset, which will allow more remaining $2 \mathrm{HDM}$ phase space to be explored.

\section{Neutral Higgs boson searches}

An additional heavy neutral Higgs boson has many decays that can be studied at the LHC. Searches are either for the direct decay products of the Higgs bosons, or for decays via other Higgs and/or gauge bosons.

ATLAS published a search for $H / A \rightarrow \tau \tau$ with the full Run 2 dataset $\left(139 \mathrm{fb}^{-1}\right)$ [11]. In the MSSM for large $\tan \beta$ the couplings to both $\tau$-leptons and $b$-type quarks are enhanced, meaning that this is a sensitive final state for that scenario. The analysis uses $\tau_{\text {lep }} \tau_{\text {had }}$ and $\tau_{\text {had }} \tau_{\text {had }}$ channels, with electron/muon and single $\tau$ triggers respectively. Each is further split into a category containing a jet originating from a $b$-quark ( $b$-tagged), and a category containing no $b$-associated jets ( $b$-veto). This is to target each of the gluon fusion and $b$-associated production modes. In addition to the larger dataset, there were improvements in the Boosted Decision Tree (BDT) to distinguish $\tau_{\text {had }}$ from jets and in the background modelling when compared to the previous $36 \mathrm{fb}^{-1}$ result [12]. Model independent limits were set on the $b$-associated and gluon fusion productions. For the MSSM, limits were set on both the hMSSM and the new $M_{h}^{125}$ scenarios, the latter is shown in Figure 1a.

ATLAS and CMS both have results with $36 \mathrm{fb}^{-1}$ for the $H \rightarrow \mu \mu$ channel [13, 14]. These search for a resonance in the observed di- $\mu$ spectrum. The analyses use a combination of a lowthreshold trigger (20-26 GeV in ATLAS, $24 \mathrm{GeV}$ in CMS) with a $\mu$ isolation requirement and a higher threshold $(50 \mathrm{GeV})$ without the isolation. Similarly to $\tau \tau$, this also favours high $\tan \beta$ in the MSSM and is split into $b$-tagged and $b$-veto channels. The ATLAS analysis uses a looser event selection than CMS in order to not bias the search to a particular signal model. When assuming a narrow resonance, ATLAS sets upper limits at $95 \%$ confidence level on the cross section times branching ratio for $b$-associated and gluon fusion between masses of $200 \mathrm{GeV}$ and $1000 \mathrm{GeV}$, with 
upper limits of $41 \mathrm{fb}$ and $1.9 \mathrm{fb}$ respectively. The CMS result extends the limits to $150 \mathrm{GeV}$ and excludes down to $20 \mathrm{fb}$ at $150 \mathrm{GeV}$ and to $0.7 \mathrm{fb}$ at $1000 \mathrm{GeV}$. The CMS analysis also includes results assuming an intrinsic width of $10 \%$ of the heavy Higgs boson mass and provides limits for the hMSSM and $m_{h}^{\text {mod }}$ scenarios.

An example of a search for Higgs boson decays to bosons is the $H \rightarrow Z A \rightarrow \ell \ell b \bar{b}$ (or $A \rightarrow Z H$ ) channel, which has results with $36 \mathrm{fb}^{-1}$ from both experiments [15, 16]. CMS presents results first assuming that $m_{H}$ is greater than $m_{A}$, but then extends the result to the opposite case. ATLAS assumes that $m_{A}$ is larger than $m_{H}$, which is motivated by the requirement for electroweak baryogenesis in $2 \mathrm{HDM}$. The search combines the clean $\ell \ell$ final state with the large $b \bar{b}$ branching ratio and selects signals based on the dijet and dilepton masses. The CMS exclusion for type-II $2 \mathrm{HDM}$ is shown in Figure 1b.

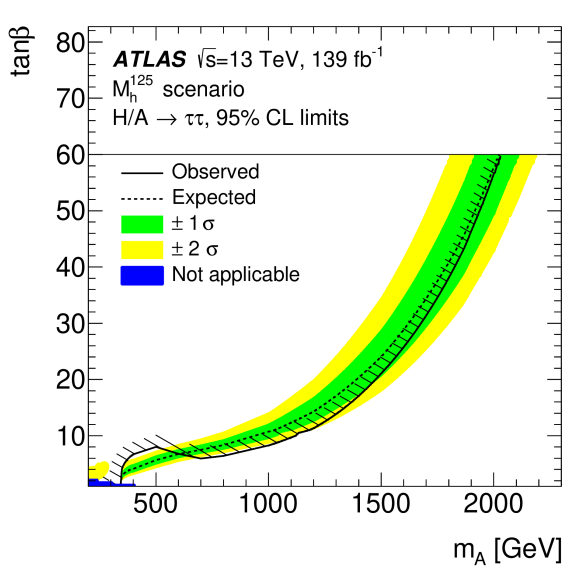

(a)

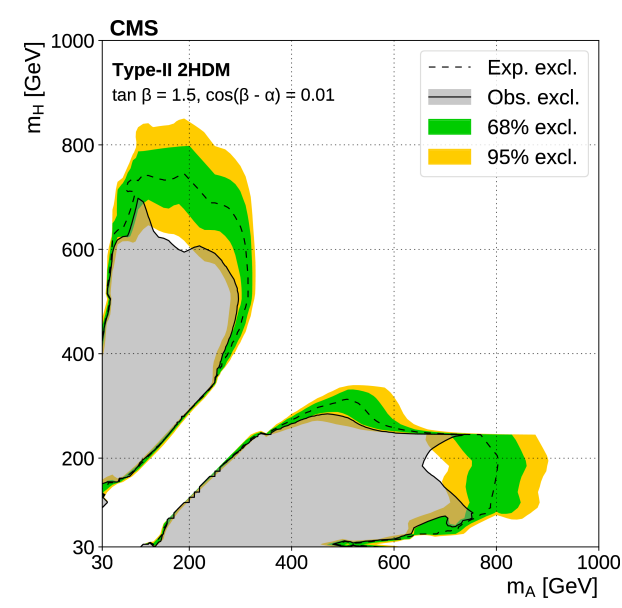

(b)

Figure 1: Selected results from searches for heavy neutral BSM Higgs bosons. (a) Exclusion from the ATLAS $H / A \rightarrow \tau \tau$ search [11] for the $M_{h}^{125}$ MSSM scenario. The blue region is where the mass splitting between the $A$ and $H$ bosons is significant when compared to the mass resolution, so the limit is not valid there. (b) Exclusion contours for type-II 2HDM with $\tan \beta=1.5$ and $\cos (\beta-\alpha)=0.01$ from the CMS $H \rightarrow Z A \rightarrow \ell \ell b \bar{b}$ analysis [16].

\section{Charged Higgs boson searches}

Any 2HDM contains only singly charged Higgs bosons, but other BSM theories can introduce doubly charged Higgs bosons. One of the main channels to search for a singly charged Higgs boson is $H^{ \pm} \rightarrow t b$. Typically the charged Higgs boson is produced in association with an additional $t b$ pair in these analyses, which leads to final states containing leptons, jets, $b$-jets, and missing transverse energy. Therefore, they are classified into several categories based on the number of jets and $b$-jets in the final state, all of which employ single lepton triggers. Both ATLAS and CMS have results with $36 \mathrm{fb}^{-1}$ of data $[17,18]$. Multi-variate analysis (MVA) techniques are employed with dedicated training in each category. Figure 2a shows the 95\% confidence level limit on the production cross section derived from the ATLAS search. In addition to $H^{ \pm} \rightarrow t b$ searches with leptons, CMS released a result in the all-hadronic final state [19]. Here, all final state objects can be reconstructed, enabling a search for an excess in the $t b$ invariant mass. A specialised jet trigger was used in this analysis. The limits are combined with the other channels and shown in Figure $2 b$. 


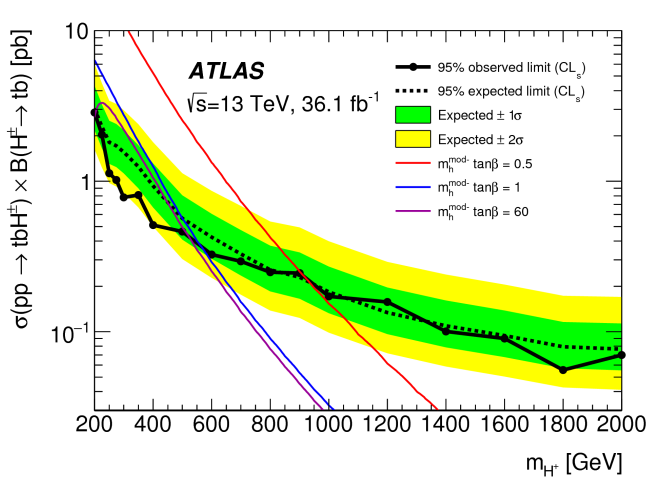

(a)

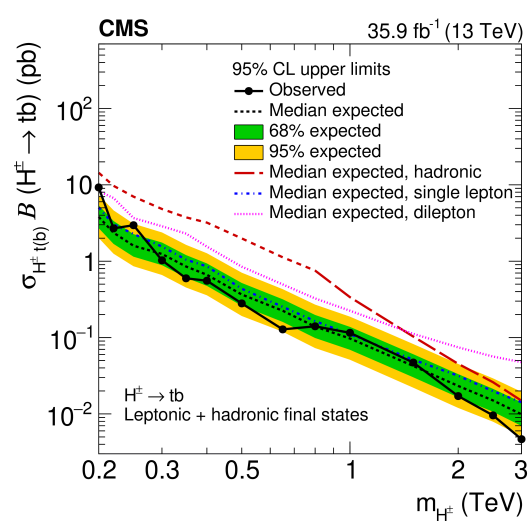

(b)

Figure 2: Limits on $H^{+} \rightarrow t b$ production in association with $t b$. (a) ATLAS limits from $\ell+$ jets and $\ell \ell$ final states [17]. Three predictions from benchmark $m_{h}^{\bmod -}$ scenarios are shown. (b) CMS upper limit from a combination of leptonic [18] and hadronic [19] final states.

Another CMS result uses a new channel to search for $H^{+} \rightarrow c s$ in the $b c s-b \ell v$ final state [20]. The invariant mass of the two non- $b$-jets is used as an observable, which is reconstructed using a kinematic fit on the reconstructed objects taking the top-quark mass as a constraint.

\section{BSM Higgs bosons in di-Higgs searches}

The Higgs self-coupling is an important aspect of the Standard Model, but the $p p \rightarrow H H$ production is predicted to have a small cross section. However, the presence of BSM physics can enhance the di-Higgs production via $g g \rightarrow X \rightarrow H H$. Therefore, analyses that set limits on the Standard Model process can also search for new BSM resonances in this channel.

The analyses generally have one Higgs boson decaying to $b \bar{b}$ thanks to its large branching ratio and combine this with another decay process. Both ATLAS and CMS have produced combinations of the different channels with $36 \mathrm{fb}^{-1}$ of integrated luminosity [21, 22]. CMS includes the $b \bar{b} \tau \tau$, $b \bar{b} \gamma \gamma, b \bar{b} W^{+} W^{-}$, and $b \bar{b} b \bar{b}$ channels, ATLAS additionally includes $W^{+} W^{-} W^{+} W^{-}$and $W^{+} W^{-} \gamma \gamma$. Upper limits on the $g g \rightarrow X \rightarrow H H$ production cross section range between $\sim 700 \mathrm{fb}$ at $300 \mathrm{GeV}$ and $\sim 0.6 \mathrm{fb}$ at $3000 \mathrm{GeV}$. These channels set competitive limits on MSSM models at low $\tan \beta$.

\section{Conclusion}

There are $36 \mathrm{fb}^{-1}$ searches for BSM Higgs bosons in many channels from both ATLAS and CMS. The full Run 2 dataset is now available, with a total of $139 \mathrm{fb}^{-1}$ and the first analyses with the complete dataset are being released. It will take some time before enough Run 3 data is available to surpass these results. Figure 3 shows the current hMSSM limits for ATLAS and $M_{h}^{125}$ limits for CMS. 


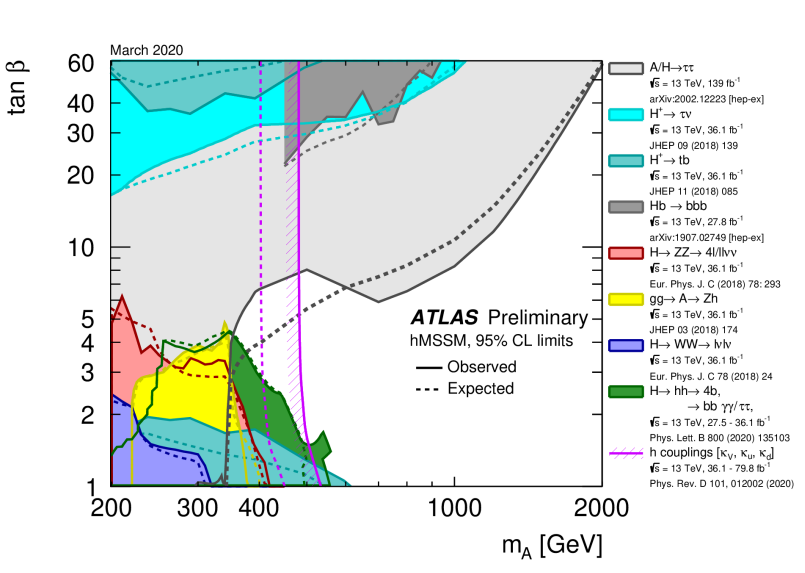

(a)

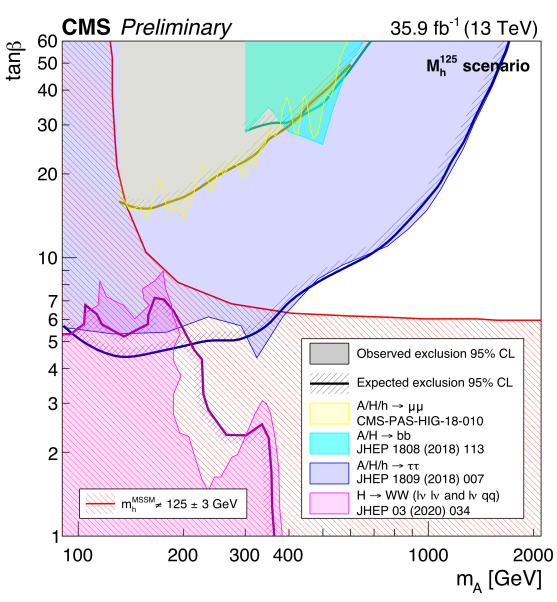

(b)

Figure 3: Excluded regions in the $\tan \beta$ versus $m_{A}$ plane for MSSM scenarios. (a) Limits from ATLAS BSM Higgs searches for the hMSSM scenario [23]. (b) CMS limits on the $M_{h}^{125}$ scenario [24].

\section{References}

[1] G. C. Branco et al., Theory and phenomenology of two-Higgs-doublet models, Phys. Rept. $516(2012) 1$.

[2] A. Djouadi, The Anatomy of electro-weak symmetry breaking. II. The Higgs bosons in the minimal supersymmetric model, Phys. Rept. 459 (2008) 1.

[3] A. Djouadi et al., The post-Higgs MSSM scenario: Habemus MSSM?, Eur. Phys. J. C 73 (2013) 2650.

[4] E. Bagnaschi et al., Benchmark scenarios for low $\tan \beta$ in the MSSM, LHCHXSWG-2015-002, (2015), available online at: http://cdsweb.cern.ch/record/2039911

[5] M. Carena, S. Heinemeyer, O. Stål, C. Wagner and G. Weiglein, MSSM Higgs boson searches at the LHC: benchmark scenarios after the discovery of a Higgs-like particle, Eur. Phys. J. C 73 (2013) 2552.

[6] E. Bagnaschi et al., MSSM Higgs boson searches at the LHC: benchmark scenarios for Run 2 and beyond, Eur. Phys. J. C 79 (2019) 617.

[7] ATLAS Collaboration, Observation of a new particle in the search for the Standard Model Higgs boson with the ATLAS detector at the LHC, Phys. Lett. B 716 (2012) 1-29.

[8] CMS Collaboration, Observation of a new boson at a mass of $125 \mathrm{GeV}$ with the CMS experiment at the LHC, Phys. Lett. B 716 (2012) 30-61.

[9] ATLAS Collaboration, The ATLAS Experiment at the CERN Large Hadron Collider, JINST 3 (2008) S08003.

[10] CMS Collaboration, The CMS experiment at the CERN LHC, JINST 3 (2008) S08004.

[11] ATLAS Collaboration, Search for Heavy Higgs Bosons Decaying into Two Tau Leptons with the ATLAS Detector Using p p Collisions at $\sqrt{s}=13 \mathrm{TeV}$, Phys. Rev. Lett. 125, (2020) 051801. 
[12] ATLAS Collaboration, Search for additional heavy neutral Higgs and gauge bosons in the ditau final state produced in $36 \mathrm{fb}^{-1}$ of pp collisions at $\sqrt{\mathrm{s}}=13 \mathrm{TeV}$ with the ATLAS detector, JHEP 01 (2018) 55.

[13] ATLAS Collaboration, Search for scalar resonances decaying into $\mu^{+} \mu^{-}$in events with and without b-tagged jets produced in proton-proton collisions at $\sqrt{s}=13 \mathrm{TeV}$ with the ATLAS detector, JHEP 07 (2019) 117.

[14] CMS Collaboration, Search for MSSM Higgs bosons decaying to $\mu^{+} \mu^{-}$in proton-proton collisions at $\sqrt{s}=13 \mathrm{TeV}$, Phys. Lett. B. 798 (2019) 134992.

[15] ATLAS Collaboration, Search for a heavy Higgs boson decaying into a Z boson and another heavy Higgs boson in the llbb final state in pp collisions at $\sqrt{s}=13 \mathrm{TeV}$ with the ATLAS detector, Phys. Lett. B 783 (2018) 392-414.

[16] CMS Collaboration, Search for new neutral Higgs bosons through the $H \rightarrow Z A \rightarrow \ell^{+} \ell^{-} b \bar{b}$ process in pp collisions $\sqrt{s}=13 \mathrm{TeV}$, JHEP 03 (2020) 55.

[17] ATLAS Collaboration, Search for charged Higgs bosons decaying into top and bottom quarks at $\sqrt{s}=13 \mathrm{TeV}$ with the ATLAS detector, JHEP 11 (2018) 85.

[18] CMS Collaboration, Search for a charged Higgs boson decaying into top and bottom quarks in events with electrons or muons in proton-proton collisions at $\sqrt{s}=13 \mathrm{TeV}$, JHEP 01 (2020) 96.

[19] CMS Collaboration, Search for charged Higgs bosons decaying into a top and a bottom quark in the all-jet final state of pp collisions at $\sqrt{s}=13 \mathrm{TeV}$, JHEP 07 (2020) 126.

[20] CMS Collaboration, Search for a light charged Higgs boson in the $H^{ \pm} \rightarrow$ cs channel in proton-proton collisions at $\sqrt{s}=13 \mathrm{TeV}$, arXiv:2005.08900, (2020) submitted to Phys. Rev. D.

[21] ATLAS Collaboration, Combination of searches for Higgs boson pairs in pp collisions at $\sqrt{s}=13$ TeV with the ATLAS detector, Phys. Lett. B 800 (2020) 135103.

[22] CMS Collaboration, Combination of Searches for Higgs Boson Pair Production in ProtonProton Collisions at $\sqrt{s}=13 \mathrm{TeV}$, Phys. Rev. Lett. 122 (2019) 121803.

[23] ATLAS Collaboration, ATLAS Higgs and DiBoson Searches Physics Group Summary Plots, available online at:

https://atlas.web.cern.ch/Atlas/GROUPS/PHYSICS/CombinedSummaryPlots/HDBS/, accessed Sept. 2020.

[24] CMS Collaboration, Higgs Physics Analysis Group Summary Plots, available online at: https://twiki.cern.ch/twiki/bin/view/CMSPublic/SummaryResultsHIG, accessed Sept. 2020. 